\title{
Korovkin type approximation theorem for functions of two variables through statistical $\boldsymbol{A}$-summability
}

\author{
Mohammad Mursaleen ${ }^{1 *}$ and Abdullah Alotaibi ${ }^{2}$
}

\section{* Correspondence:}

mursaleenm@gmail.com

'Department of Mathematics,

Aligarh Muslim University, Aligarh 202002, India

Full list of author information is

available at the end of the article

\section{Abstract}

In this article, we prove a Korovkin type approximation theorem for a function of two variables by using the notion of statistical $A$-summability. We also study the rate of statistical A-summability of positive linear operators. Finally we construct an example by Bleimann et al. operators to show that our result is stronger than those of previously proved by other authors.

AMS Subject Classification 2000: 41A10; 41A25; 41A36; 40A30; 40G15.

Keywords: density, statistical convergence, A-statistical convergence, statistical Asummability, positive linear operator, Korovkin type approximation theorem

\section{Introduction and preliminaries}

The concept of statistical convergence for sequences of real numbers was introduced by Fast [1] and further studied many others.

Let $K \subseteq \mathbb{N}$ and $K_{n}=\{k \leq n: k \in K\}$. Then the natural density of $K$ is defined by $\delta(K)=$ $\lim _{n} n^{-1}\left|K_{n}\right|$ if the limit exists, where $\left|K_{n}\right|$ denotes the cardinality of $K_{n}$.

A sequence $x=\left(x_{k}\right)$ of real numbers is said to be statistically convergent to $L$ provided that for every $\varepsilon>0$ the set $K_{\varepsilon}:=\left\{k \in \mathbb{N}:\left|x_{\mathrm{k}}-L\right| \geq \varepsilon\right\}$ has natural density zero, i.e. for each $\varepsilon>0$,

$$
\lim _{n} \frac{1}{n}\left|\left\{k \leq n:\left|x_{k}-L\right| \geq \varepsilon\right\}\right|=0 .
$$

In this case we write $s t$ - $\lim x=L$. Note that if $x=\left(x_{k}\right)$ is convergent then it is statistically convergent but not conversely. The idea of statistical convergence of double sequences has been intruduced and studied in $[2,3]$.

Let $A=\left(a_{n k}\right), n, k \in \mathbb{N}$, be an infinite matrix and $x=\left(x_{k}\right)$ be a sequence. Then the (transformed) sequence, $A x:=\left(y_{n}\right)$, is denoted by

$$
y_{n}:=\sum_{k=1}^{\infty} a_{n k} x_{k \prime}
$$

where it is assumed that the series on the right converges for each $n \in \mathbb{N}$. We say that a sequence $x$ is $A$-summable to the limit $\ell$ if $y_{n} \rightarrow \ell$ as $n \rightarrow \infty$.

A matrix transformation is said to be regular if it maps every convergent sequence into a convergent sequence with the same limit. The well-known conditions for two dimensional matrix to be regular are known as Silverman-Toeplitz conditions.

(C) 2012 Mursaleen and Alotaibi; licensee Springer. This is an Open Access article distributed under the terms of the Creative Commons Attribution License (http://creativecommons.org/licenses/by/2.0), which permits unrestricted use, distribution, and reproduction in any medium, provided the original work is properly cited. 
In [4], Edely and Mursaleen have given the notion of statistical $A$-summability for single sequences and statistical $A$-summability for double sequences has recently been studied in [5].

Let $A=\left(a_{n k}\right)$ be a nonnegative regular summability matrix and $x=\left(x_{k}\right)$ be a sequence of real or complex sequences. We say that $x$ is statistically $A$-summable to $L$ if for every $\varepsilon>0$,

$$
\delta\left(\left\{n \in \mathbb{N}:\left|\gamma_{n}-L\right| \geq \varepsilon\right\}\right)=0 .
$$

So, if $x$ is statistically $A$-summable to $L$ then for every $\varepsilon>0$,

$$
\lim _{m} \frac{1}{m}\left|\left\{n \leq m:\left|\gamma_{n}-L\right| \geq \varepsilon\right\}\right|=0 .
$$

Note that if a sequence is bounded and $A$-statistically convergent to $L$, then it is $A$ summable to $L$; hence it is statistically $A$-summable to $L$ but not conversely (see [4]).

Example 1.1. Let $A=(C, 1)$, the Cesàro matrix and the sequence $u=\left(u_{k}\right)$ be defined by

$$
u_{k}= \begin{cases}1 & \text { if } \mathrm{k} \text { is odd } \\ 0 & \text { if } \mathrm{k} \text { is even }\end{cases}
$$

Then $u$ is is $A$-summable to $\frac{1}{2}$ (and hence statistically $A$-summable to $1 / 2$ ) but not statistically (and not $A$-statistically as well) convergent.

Let $I:=[0, \infty)$ and $C(I)$ denote the space of all continuous real valued functions on $I$. Let $C_{B}(I):=\{f \in C(I): f$ is bounded on $I\} . C(I)$ and $C_{B}(I)$ are equipped with norm

$$
\|f\|_{C(I)}:=\sup _{x \in I}|f(x)| .
$$

Let $H_{\omega}(I)$ denote the space of all real valued functions $f$ on $I$ such that

$$
|f(s)-f(x)| \leq \omega\left(f ;\left|\frac{s}{1+s}-\frac{x}{1+x}\right|\right),
$$

where $\omega$ is the modulus of continuity, i.e.

$$
\omega(f ; \delta)=\sup _{s, x \in I}\{|f(s)-f(x)|:|s-x| \leq \delta\} .
$$

It is to be noted that any function $f \in H_{\omega}(I)$ is continuous and bounded on $I$.

The following Korovkin type theorem (see [6]) was proved by Çakar and Gadjiev [7].

Theorem A. Let $\left(L_{n}\right)$ be a sequence of positive linear operators from $H_{\omega}(I)$ into $C_{B}$ (I). Then for all $f \in H_{\omega}(I)$

$$
\lim _{n \rightarrow \infty}\left\|L_{n}(f ; x)-f(x)\right\|_{C_{B}(I)}=0
$$

if and only if

$$
\lim _{n \rightarrow \infty}\left\|L_{n}\left(f_{i} ; x\right)-g_{i}\right\|_{C_{B}(I)}=0(i=0,1,2),
$$

where

$$
g_{0}(x)=1, g_{1}(x)=\frac{x}{1+x}, g_{2}(x)=\left(\frac{x}{1+x}\right)^{2} .
$$


Erkuş and Duman [8] have given the $s t_{A}$-version of the above theorem for functions of two variables.Quite recently, Korovkin type of approximation theorems have been proved in $[9,10]$ by using almost convergence; in [11-15] by using variants of statistical convergence and in [16-19] for functions of two variables by using statistical convergence, $A$-statistical convergence and statistical $A$-summability of double sequences. In this article, we use the notion of statistical $A$-summability to prove a Korovkin type approximation theorem for functions of two variables with the help of test functions $1, \frac{x}{1+x}, \frac{y}{1+y},\left(\frac{x}{1+x}\right)^{2}+\left(\frac{y}{1+y}\right)^{2}$.

\section{Main result}

Let $I=[0, \infty)$ and $K=I \times I$. We denote by $C_{B}(K)$ the space of all bounded and continuous real valued functions on $K$ equipped with norm

$$
\|f\|_{C_{B}(K)}:=\sup _{(x, y) \in K}|f(x, y)|, \quad f \in C_{B}(K) .
$$

Let $H_{\omega^{*}}(K)$ denote the space of all real valued functions $f$ on $K$ such that

$$
|f(s, t)-f(x, y)| \leq \omega^{*}\left(f ; \sqrt{\left(\frac{s}{1+s}-\frac{x}{1+x}\right)^{2}+\left(\frac{t}{1+t}-\frac{y}{1+y}\right)^{2}}\right),
$$

where $\omega^{*}$ is the modulus of continuity, i.e.

$$
\omega^{*}(f ; \delta)=\sup _{(s, t),(x, y) \in K}\left\{|f(s, t)-f(x, y)|: \sqrt{(s-x)^{2}+(t-y)^{2}} \leq \delta\right\} .
$$

It is to be noted that any function $f \in H_{\omega^{*}}(K)$ is bounded and continuous on $K$, and a necessary and sufficient condition for $f \in H_{\omega^{\prime \prime}}(K)$ is that

$$
\lim _{\delta \rightarrow 0} \omega^{*}(f ; \delta)=0 .
$$

We prove the following result:

Theorem 2.1. Let $A=\left(a_{n k}\right)$ be nonnegative regular summability matrix. Let $\left(T_{k}\right)$ be a sequence of positive linear operators from $H_{\omega^{*}}(K)$ into $C_{B}(K)$. Then for all $f \in H_{\omega^{*}}(K)$

$$
s t-\lim _{n \rightarrow \infty}\left\|\sum_{k=1}^{\infty} a_{n k} T_{k}(f ; x, y)-f(x, y)\right\|_{C_{B}(K)}=0
$$

if and only if

$$
\begin{aligned}
& s t-\lim _{n \rightarrow \infty}\left\|\sum_{k=1}^{\infty} a_{n k} T_{k}(1 ; x, y)-1\right\|_{C_{B}(K)}=0 \\
& \text { st }-\lim _{n \rightarrow \infty}\left\|\sum_{k=1}^{\infty} a_{n k} T_{k}\left(\frac{s}{1+s} ; x, y\right)-\frac{x}{1+x}\right\|_{C_{B}(K)}=0 \\
& \text { st }-\lim _{n \rightarrow \infty}\left\|\sum_{k=1}^{\infty} a_{n k} T_{k}\left(\frac{t}{1+t} ; x, y\right)-\frac{y}{1+y}\right\|_{C_{B}(K)}=0
\end{aligned}
$$




$$
\text { st }-\lim _{n \rightarrow \infty}\left\|\sum_{k=1}^{\infty} a_{n k} T_{k}\left(\left(\frac{s}{1+s}\right)^{2}+\left(\frac{t}{1+t}\right)^{2} ; x, y\right)-\left(\left(\frac{x}{1+x}\right)^{2}+\left(\frac{y}{1+\gamma}\right)^{2}\right)\right\|_{C_{B}(K)}=0
$$

Proof. Since of the functions $f_{0}(x, y)=1, f_{1}(x, y)=\frac{x}{1+x}, f_{2}(x, y)=\frac{y}{1+y}, f_{3}(x, y)=\left(\frac{x}{1+x}\right)^{2}+\left(\frac{y}{1+y}\right)^{2}$ belongs to $H_{\omega^{* *}}(K)$, conditions (2.1)-(2.4) follow immediately from (2.0).

Let $f \in H_{\omega^{\prime \prime}}(K)$ and $(x, y) \in K$ be fixed. Then for $\varepsilon>0$ there exist $\delta_{1}, \delta_{2}>0$ such that $|f(s, t)-f(x, y)|<\varepsilon$ holds for all $(s, t) \in K$ satisfying $\left|\frac{s}{1+s}-\frac{x}{1+x}\right|<\delta_{1}$ and $\left|\frac{t}{1+t}-\frac{y}{1+y}\right|<\delta_{2}$.

Let

$$
K(\delta):=\left\{(s, t) \in K: \sqrt{\left(\frac{s}{1+s}-\frac{x}{1+x}\right)^{2}+\left(\frac{t}{1+t}-\frac{\gamma}{1+\gamma}\right)^{2}}<\delta=\min \left\{\delta_{1}, \delta_{2}\right\}\right\} .
$$

Hence

$$
\begin{aligned}
|f(s, t)-f(x, y)|=\mid f(s, t) & -f(x, y)\left|\chi_{K(\delta)}(s, t)+\right| f(s, t)-f(x, y) \mid \chi_{K \backslash K(\delta)}(s, t) \\
& \leq \varepsilon+2 N \chi_{K \backslash K(\delta)}(s, t)
\end{aligned}
$$

Where $\chi_{D}$ denotes the characteristic function of the set $D$ and $N=\|f\|_{C_{B}(K)}$. Further we get

$$
\chi_{K \backslash K(\delta)}(s, t) \leq \frac{1}{\delta_{1}^{2}}\left(\frac{s}{1+s}-\frac{x}{1+x}\right)^{2}+\frac{1}{\delta_{2}^{2}}\left(\frac{t}{1+t}-\frac{y}{1+y}\right)^{2} .
$$

Combining (2.5) and (2.6), we get

$$
|f(s, t)-f(x, y)| \leq \varepsilon+\frac{2 N}{\delta^{2}}\left\{\left(\frac{s}{1+s}-\frac{x}{1+x}\right)^{2}+\left(\frac{t}{1+t}-\frac{y}{1+y}\right)^{2}\right\},
$$

After using the properties of $f$, a simple calculation gives that

$$
\begin{gathered}
\left|T_{k}(f ; x, y)-f(x, y)\right| \leq \varepsilon+M\left\{\left|T_{k}\left(f_{0} ; x, y\right)-f_{0}(x, y)\right|+\left|T_{k}\left(f_{1} ; x, y\right)-f_{1}(x, y)\right|\right. \\
\left.+\left|T_{k}\left(f_{2} ; x, y\right)-f_{2}(x, y)\right|+\left|T_{k}\left(f_{3} ; x, y\right)-f_{3}(x, y)\right|\right\},
\end{gathered}
$$

where

$$
M:=\varepsilon+N+\frac{4 N}{\delta^{2}} .
$$

Now replacing $T_{k}(f ; x, y)$ by $\sum_{k=1}^{\infty} a_{n k} T_{k}(f ; x, y)$ and taking $\sup _{(x, y) \in K}$, we get

$$
\begin{gathered}
\left\|\sum_{k=1}^{\infty} a_{n k} T_{k}(f ; x, y)-f(x, y)\right\|_{C_{B}(K)} \leq \varepsilon+M\left(\left\|\sum_{k=1}^{\infty} a_{n k} T_{k}\left(f_{0} ; x, y\right)-f_{0}(x, y)\right\|_{C_{B}(K)}\right. \\
+\left\|\sum_{k=1}^{\infty} a_{n k} T_{k}\left(f_{1} ; x, y\right)-f_{1}(x, y)\right\|_{C_{B}(K)}+\left\|\sum_{k=1}^{\infty} a_{n k} T_{k}\left(f_{2} ; x, y\right)-f_{2}(x, y)\right\|_{C_{B}(K)} \\
\left.+\left\|\sum_{k=1}^{\infty} a_{n k} T_{k}\left(f_{3} ; x, y\right)-f_{3}(x, y)\right\|_{C_{B}(K)}\right) .
\end{gathered}
$$


For a given $r>0$ choose $\varepsilon>0$ such that $\varepsilon<r$. Define the following sets

$$
\begin{aligned}
& D:=\left\{n:\left\|\sum_{k=1}^{\infty} a_{n k} T_{k}(f ; x, y)-f(x, y)\right\|_{C_{B}(K)} \geq r\right\}, \\
& D_{1}:=\left\{n:\left\|\sum_{k=1}^{\infty} a_{n k} T_{k}\left(f_{0} ; x, y\right)-f_{0}(x, y)\right\|_{C_{B}(K)} \geq \frac{r-\varepsilon}{4 K}\right\}, \\
& D_{2}:=\left\{n:\left\|\sum_{k=1}^{\infty} a_{n k} T_{k}\left(f_{1} ; x, y\right)-f_{1}(x, y)\right\|_{C_{B}(K)} \geq \frac{r-\varepsilon}{4 K}\right\}, \\
& D_{3}:=\left\{n:\left\|\sum_{k=1}^{\infty} a_{n k} T_{k}\left(f_{2} ; x, y\right)-f_{2}(x, y)\right\|_{C_{B}(K)} \geq \frac{r-\varepsilon}{4 K}\right\}, \\
& D_{4}:=\left\{n:\left\|\sum_{k=1}^{\infty} a_{n k} T_{k}\left(f_{3} ; x, y\right)-f_{3}(x, y)\right\|_{C_{B}(K)} \geq \frac{r-\varepsilon}{4 K}\right\} .
\end{aligned}
$$

Then from (2.9), we see that $D \subset D_{1} \cup D_{2} \cup D_{3} \cup D_{4}$ and therefore $\delta(D) \leq \delta\left(D_{1}\right)+$ $\delta\left(D_{2}\right)+\delta\left(D_{3}\right)+\delta\left(D_{4}\right)$. Hence conditions (2.1)-(2.4) imply the condition (2.0).

This completes the proof of the theorem.

If we replace the matrix $A$ in Theorem 2.1 by identity matrix, then we immediately get the following result which is due to Erkuş and Duman [8]:

Corollay 2.2. Let $A=\left(a_{n k}\right)$ be nonnegative regular summability matrix. Let $\left(T_{k}\right)$ be a sequence of positive linear operators from $H_{\omega^{*}}(K)$ into $C_{B}(K)$. Then for all $f \in H_{\omega^{*}}(K)$

$$
\text { st - } \lim _{k \rightarrow \infty}\left\|T_{k}(f ; x, y)-f(x, y)\right\|_{C_{B}(K)}=0
$$

if and only if

$$
\begin{aligned}
& \text { st }-\lim _{k \rightarrow \infty}\left\|T_{k}(1 ; x, y)-1\right\|_{C_{B}(K)}=0, \\
& \text { st }-\lim _{k \rightarrow \infty}\left\|T_{k}\left(\frac{s}{1+s} ; x, y\right)-\frac{x}{1+x}\right\|_{C_{B}(K)}=0, \\
& \text { st }-\lim _{k \rightarrow \infty}\left\|T_{k}\left(\frac{t}{1+t} ; x, y\right)-\frac{y}{1+y}\right\|_{C_{B}(K)}=0, \\
& \text { st }-\lim _{k \rightarrow \infty}\left\|T_{k}\left(\left(\frac{s}{1+s}\right)^{2}+\left(\frac{t}{1+t}\right)^{2} ; x, y\right)-\left(\left(\frac{x}{1+x}\right)^{2}+\left(\frac{y}{1+y}\right)^{2}\right)\right\|_{C_{B}(K)}=0 .
\end{aligned}
$$

\section{Statistical rate of convergence}

In this section, using the concept of statistical $A$-summability we study the rate of convergence of positive linear operators with the help of the modulus of continuity. Let us recall, for $f \in H_{\omega^{*}}(K)$

$$
|f(s, t)-f(x, y)| \leq \omega^{*}\left(f ; \sqrt{\left(\frac{s}{1+s}-\frac{x}{1+x}\right)^{2}+\left(\frac{t}{1+t}-\frac{y}{1+y}\right)^{2}}\right),
$$


where

$$
\omega^{*}(f ; \delta)=\sup _{(s, t),(x, y) \in K}\left\{|f(s, t)-f(x, y)|: \sqrt{(s-x)^{2}+(t-y)^{2}} \leq \delta\right\} .
$$

We have the following result:

Theorem 3.1. Let $A=\left(a_{n k}\right)$ be nonnegative regular summability matrix. Let $\left(T_{k}\right)$ be a sequence of positive linear operators from $H_{\omega^{*}}(K)$ into $C_{B}(K)$. Assume that

(i) st $-\lim _{n \rightarrow \infty}\left\|\sum_{k=1}^{\infty} a_{n k} T_{k}\left(f_{0}\right)-f_{0}\right\|_{C_{B}(K)}=0$,

(ii) st- $\lim _{n \rightarrow 0} \omega^{*}\left(f ; \delta_{n}\right)=0$, where

$$
\delta_{n}=\sqrt{\left\|\sum_{k=1}^{\infty} a_{n k} T_{k}(\psi)\right\|_{C_{B}(K)}} \text { with } \psi=\psi(s, t)=\left(\frac{s}{1+s}-\frac{x}{1+x}\right)^{2}+\left(\frac{t}{1+t}-\frac{y}{1+y}\right)^{2} .
$$

Then for all $f \in H_{\omega^{*}}(K)$

$$
\text { st }-\lim _{n \rightarrow \infty}\left\|\sum_{k=1}^{\infty} a_{n k} T_{k}(f)-f\right\|_{C_{B}(K)}=0 .
$$

Proof. Let $f \in H_{\omega^{*}}(K)$ be fixed and $(x, y) \in K$ be fixed. Using linearity and positivity of the operators $T_{k}$ for all $n \in \mathbb{N}$, we have

$$
\begin{aligned}
& \left|\sum_{k=1}^{\infty} a_{n k} T_{k}(f ; x, y)-f(x, y)\right| \leq \sum_{k=1}^{\infty} a_{n k} T_{k}(|f(s, t)-f(x, y)| ; x, y) \\
& +|f(x, y)|\left|\sum_{k=1}^{\infty} a_{n k} T_{k}\left(f_{0} ; x, y\right)-f_{0}(x, y)\right| \\
& \leq \sum_{k=1}^{\infty} a_{n k} T_{k}\left(\omega^{*}\left(f ; \delta \frac{\sqrt{\left(\frac{s}{1+s}-\frac{x}{1+x}\right)^{2}+\left(\frac{t}{1+t}-\frac{\gamma}{1+\gamma}\right)^{2}}}{\delta}\right) ; x, y\right) \\
& +\|f\|_{C_{B}(K)}\left|\sum_{k=1}^{\infty} a_{n k} T_{k}\left(f_{0} ; x, y\right)-f_{0}(x, y)\right| \\
& \leq \sum_{k=1}^{\infty} a_{n k} T_{k}\left(\left(1+\left[\frac{\sqrt{\left(\frac{s}{1+s}-\frac{x}{1+x}\right)^{2}+\left(\frac{t}{1+t}-\frac{y}{1+y}\right)^{2}}}{\delta}\right]\right) \omega^{*}(f ; \delta) ; x, y\right) \\
& +\|f\|_{C_{B}(K)}\left|\sum_{k=1}^{\infty} a_{n k} T_{k}\left(f_{0} ; x, y\right)-f_{0}(x, y)\right| \\
& \leq \sum_{k=1}^{\infty} a_{n k} \omega^{*}(f ; \delta) T_{k}\left(\left(1+\frac{\left(\frac{s}{1+s}-\frac{x}{1+x}\right)^{2}+\left(\frac{t}{1+t}-\frac{\gamma}{1+\gamma}\right)^{2}}{\delta^{2}}\right) ; x, y\right) \\
& +\|f\|_{C_{B}(K)}\left|\sum_{k=1}^{\infty} a_{n k} T_{k}\left(f_{0} ; x, y\right)-f_{0}(x, y)\right| \\
& \leq \omega^{*}(f ; \delta)\left|\sum_{k=1}^{\infty} a_{n k} T_{k}\left(f_{0} ; x, y\right)-f_{0}(x, y)\right|+|| f \|_{C_{B}(K)}\left|\sum_{k=1}^{\infty} a_{n k} T_{k}\left(f_{0} ; x, y\right)-f_{0}(x, y)\right| \\
& +\omega^{*}(f ; \delta)+\frac{\omega^{*}(f ; \delta)}{\delta^{2}} \sum_{k=1}^{\infty} a_{n k} T_{k}\left(\left(\left(\frac{s}{1+s}-\frac{x}{1+x}\right)^{2}+\left(\frac{t}{1+t}-\frac{y}{1+y}\right)^{2} ; x, y\right)\right) \text {. }
\end{aligned}
$$


Hence

$$
\begin{gathered}
\left\|\sum_{k=1}^{\infty} a_{n k} T_{k}(f)-f\right\|_{C_{B}(K)} . \\
\leq\|f\|_{C_{B}(K)}\left\|\sum_{k=1}^{\infty} a_{n k} T_{k}\left(f_{0}\right)-f_{0}\right\|_{C_{B}(K)}+\omega^{*}(f ; \delta)\left\|\sum_{k=1}^{\infty} a_{n k} T_{k}\left(f_{0}\right)-f_{0}\right\|_{C_{B}(K)} \\
+\frac{\omega^{*}(f ; \delta)}{\delta^{2}}\left\|\sum_{k=1}^{\infty} a_{n k} T_{k}(\psi)\right\|_{C_{B}(K)}+\omega^{*}(f ; \delta) .
\end{gathered}
$$

Now if we choose $\delta:=\delta_{n}:=\sqrt{\left\|\sum_{k=1}^{\infty} a_{n k} T_{k}(\psi)\right\|_{C_{B}(K)}}$ then

$$
\begin{gathered}
\left\|\sum_{k=1}^{\infty} a_{n k} T_{k}(f)-f\right\|_{C_{B}(K)} . \\
\leq\|f\|_{C_{B}(K)}\left\|\sum_{k=1}^{\infty} a_{n k} T_{k}\left(f_{0}\right)-f_{0}\right\|_{C_{B}(K)} \\
+\omega^{*}\left(f ; \delta_{n}\right)\left\|\sum_{k=1}^{\infty} a_{n k} T_{k}\left(f_{0}\right)-f_{0}\right\|_{C_{B}(K)}+2 \omega^{*}\left(f ; \delta_{n}\right) .
\end{gathered}
$$

Therefore

$$
\begin{gathered}
\left\|\sum_{k=1}^{\infty} a_{n k} T_{k}(f)-f\right\|_{C_{B}(K)} . \\
\leq M\left\{\left\|\sum_{k=1}^{\infty} a_{n k} T_{k}\left(f_{0}\right)-f_{0}\right\|_{C_{B}(K)}\right. \\
\left.+\omega^{*}\left(f ; \delta_{n}\right)\left\|\sum_{k=1}^{\infty} a_{n k} T_{k}\left(f_{0}\right)-f_{0}\right\|_{C_{B}(K)}+\omega^{*}\left(f ; \delta_{n}\right)\right\},
\end{gathered}
$$

where $M=\max \left\{2,\|f\|_{C_{B}(K)}\right\}$. Now, for a given $r>0$, choose $\varepsilon>0$ such that $\varepsilon>r$. Let us write

$$
\begin{aligned}
& E:\left\{n:\left\|\sum_{k=1}^{\infty} a_{n k} T_{k}(f ; x, y)-f(x, y)\right\|_{C_{B}(K)} \geq r\right\}, \\
& E_{1}:=\left\{n:\left\|\sum_{k=1}^{\infty} a_{n k} T_{k}\left(f_{0} ; x, y\right)-f_{0}(x, y)\right\|_{C_{B}(K)} \geq \frac{r}{3 K}\right\}, \\
& E_{2}:=\left\{n: \omega^{*}\left(f ; \delta_{n}\right) \geq \frac{r}{3 K}\right\}, \\
& E_{3}:=\left\{n: \omega^{*}\left(f ; \delta_{n}\right)\left\|\sum_{k=1}^{\infty} a_{n k} T_{k}\left(f_{0} ; x, y\right)-f_{0}(x, y)\right\|_{C_{B}(K)} \geq \frac{r}{3 K}\right\} .
\end{aligned}
$$

Then $E \subset E_{1} \cup E_{2} \cup E_{3}$ and therefore $\delta(E) \leq \delta\left(E_{1}\right)+\delta\left(E_{2}\right)+\delta\left(E_{3}\right)$. Using conditions (i) and (ii) we conclude 


$$
\text { st }-\lim _{n \rightarrow \infty}\left\|\sum_{k=1}^{\infty} a_{n k} T_{k}(f)-f\right\|_{C_{B}(K)}=0 .
$$

This completes the proof of the theorem.

\section{Example and the concluding remark}

We show that the following double sequence of positive linear operators satisfies the conditions of Theorem 2.1 but does not satisfy the conditions of Corollary 2.2 and Theorem 2.1 of [8].

Example 4.1. Consider the following Bleimann et al. [20] (of two variables) operators:

$$
B_{n}(f ; x, y):=\frac{1}{(1+x)^{n}(1+y)^{n}} \sum_{j=0}^{n} \sum_{k=0}^{n} f\left(\frac{j}{n-j+1}, \frac{k}{n-k+1}\right)\left(\begin{array}{l}
n \\
j
\end{array}\right)\left(\begin{array}{l}
n \\
k
\end{array}\right) x^{j} y^{k},
$$

where $f \in H_{\omega}(K), K=[0, \infty) \times[0, \infty)$ and $n \in \mathbb{N}$.

Since

$$
(1+x)^{n}=\sum_{j=0}^{n}\left(\begin{array}{c}
m \\
j
\end{array}\right) x^{j}
$$

it is easy to see that

$$
B_{n}\left(f_{0} ; x, y\right) \rightarrow 1=f_{0}(x, y) .
$$

Also by simple calculation, we obtain

$$
B_{n}\left(f_{1} ; x, y\right)=\frac{n}{n+1}\left(\frac{x}{1+x}\right) \rightarrow \frac{x}{1+x}=f_{1}(x, y),
$$

and

$$
B_{n}\left(f_{2} ; x, y\right)=\frac{n}{n+1}\left(\frac{y}{1+y}\right) \rightarrow \frac{y}{1+y}=f_{2}(x, y) .
$$

Finally, we get

$$
\begin{aligned}
\quad B_{n}\left(f_{3} ; x, y\right) & \frac{n(n-1)}{(n+1)^{2}}\left(\frac{x}{1+x}\right)^{2}+\frac{n}{(n+1)^{2}}\left(\frac{x}{1+x}\right) \\
& +\frac{n(n-1)}{(n+1)^{2}}\left(\frac{y}{1+y}\right)^{2}+\frac{n}{(n+1)^{2}}\left(\frac{y}{1+y}\right) \\
\rightarrow & \left(\frac{x}{1+x}\right)^{2}+\left(\frac{y}{1+y}\right)^{2}=f_{3}(x, y) .
\end{aligned}
$$

Now, take $A=C(1,1)$ and define $u=\left(u_{n}\right)$ by (1.1). Let the operator $L_{n}: H_{\omega}(K) \rightarrow C_{B}$ $(K)$ be defined by

$$
L_{n}(f ; x, y)=\left(1+u_{n}\right) B_{n}(f ; x, y) .
$$

It is easy to see that the sequence $\left(L_{n}\right)$ satisfies the conditions (2.1)-(2.4). Hence by Theorem 2.1, we have 


$$
\begin{aligned}
& s t- \lim _{m \rightarrow \infty}\left\|\sum_{n=1}^{\infty} a_{m n} L_{n}(f ; x, y)-f(x, y)\right\|_{C_{B}(K)} \\
&=s t-\lim _{m \rightarrow \infty}\left\|\frac{1}{m} \sum_{n=1}^{m} L_{n}(f ; x, y)-f(x, y)\right\|_{C_{B}(K)}=0 .
\end{aligned}
$$

On the other hand, the sequence $\left(L_{n}\right)$ does not satisfy the conditions of Theorem A and Corollary 2.2 and Theorem 2.1 of [8], since $\left(L_{n}\right)$ is neither convergent nor statistically (nor A-statistically) convergent. That is, Theorem A, Corollary 2.2 and Theorem 2.1 of [8] do not work for our operators $L_{n}$. Hence our Theorem 2.1 is stronger than Corollary 2.2 and Theorem 2.1 of [8].

\section{Author details}

${ }^{1}$ Department of Mathematics, Aligarh Muslim University, Aligarh 202002, India ²Department of Mathematics, King Abdulaziz University, Jeddah 21589, Kingdom of Saudi Arabia

\section{Authors' contributions}

The main result of the paper was proposed and proved by AA, Section 3 was given by MM, and Section 4 was designed by both the authors jointly. All authors read and approved the final manuscript.

\section{Competing interests}

The authors declare that they have no competing interests.

Received: 13 March 2012 Accepted: 23 May 2012 Published: 23 May 2012

\section{References}

1. Fast, H: Sur la convergence statistique. Collog Math. 2, 241-244 (1951)

2. Moricz, F: Statistical convergence of multiple sequences. Arch Math. 81, 82-89 (2003). doi:10.1007/s00013-003-0506-9

3. Mursaleen, M, Edely, OHH: Statistical convergence of double sequences. J Math Anal Appl. 288, 223-231 (2003). doi:10.1016/j.jmaa.2003.08.004

4. Edely, $\mathrm{OHH}$, Mursaleen, M: On statistical A-summability. Math Comput Model. 49, 672-680 (2009). doi:10.1016/j. mcm.2008.05.053

5. Belen, C, Mursaleen, M, Yildirim, M: Statistical A-summability of double sequences and a Korovkin type approximation theorem. Bull Korean Math Soc. 49(4), 851-861 (2012)

6. Korovkin, PP: Linear Operators And Approximation Theory. Hindustan Publ. Co., Delhi (1960)

7. Cakar, Ö, Gadjiev, AD: On uniform approximation by Bleimann, Butzer and Hahn on all positive semiaxis. Tras Acad Sci Azerb Ser Phys Tech Math Sci. 19, 21-26 (1999)

8. ErkuÅŸ, E, Duman, O: A-Statistical extension of the Korovkin type approximation theorem. Proc Indian Acad Sci (Math Sci). 115(4), 499-508 (2005). doi:10.1007/BF02829812

9. Anastassiou, GA, Mursaleen, M, Mohiuddine, SA: Some approximation theorems for functions of two variables through almost convergence of double sequences. J Comput Anal Appl. 13, 37-40 (2011)

10. Mohiuddine, SA: An application of almost convergence in approximation theorems. Appl Math Lett. 24, 1856-1860 (2011). doi:10.1016/j.aml.2011.05.006

11. Edely, $\mathrm{OHH}$, Mohiuddine, SA, Noman, AK: Korovkin type approximation theorems obtained through generalized statistical convergence. Appl Math Lett. 23, 1382-1387 (2010). doi:10.1016/j.aml.2010.07.004

12. Mursaleen, M, Alotaibi, A: Statistical summability and approximation by de la Vallée-Poussin mean. Appl Math Lett. 24 320-324 (2011). doi:10.1016/j.aml.2010.10.014

13. Mursaleen, M, Alotaibi, A: Statistical lacunary summability and a Korovkin type approximation theorem. Ann Univ Ferrara. 57(2), 373-381 (2011). doi:10.1007/s11565-011-0122-8

14. Mursaleen, M, Karakaya, V, Ertürk, M, Gürsoy, F: Weighted statistical convergence and its application to Korovkin type approximation theorem. Appl Math Comput. 218, 9132-9137 (2012). doi:10.1016/j.amc.2012.02.068

15. Srivastava, HM, Mursaleen, M, Khan, A: Generalized equi-statistical convergence of positive linear operators and associated approximation theorems. Math Comput Mod. 55, 2040-2051 (2012). doi:10.1016/j.mcm.2011.12.011

16. Demircim, K, KarakuÅŸ, S: Statistical A-summability of positive linear operators. Math Comput Model. 53, 189-195 (2011). doi:10.1016/j.mcm.2010.08.003

17. Demirci, K, KarakuÅŸ, S: Korovkin-type approximation theorem for double sequences of positive linear operators via statistical A-summability. Results Math (2011). doi:10.1007/s00025-011-0140-y

18. Dirik, F, Demirci, K: Korovkin type approximation theorem for functions of two variables in statistical sense. Turk J Math. 34, 73-83 (2010)

19. Dirik, F, Demirci, K: A Korovkin type approximation theorem for double sequences of positive linesr operators of two variables in A-statistical sense. Bull Korean Math Soc. 47, 825-837 (2010). doi:10.4134/BKMS.2010.47.4.825 
20. Bleimann, G, Butzer, PL, Hahn, L: A Bernstein type operator approximating continuous functions on semiaxis. Indag Math. 42, 255-262 (1980)

doi:10.1186/1687-1847-2012-65

Cite this article as: Mursaleen and Alotaibi: Korovkin type approximation theorem for functions of two variables

through statistical $\boldsymbol{A}$-summability. Advances in Difference Equations 2012 2012:65.

Submit your manuscript to a SpringerOpen ${ }^{\odot}$ journal and benefit from:

- Convenient online submission

- Rigorous peer review

- Immediate publication on acceptance

- Open access: articles freely available online

- High visibility within the field

- Retaining the copyright to your article

Submit your next manuscript at $\boldsymbol{\nabla}$ springeropen.com 